\title{
Air Pollution: The Environmental Phoenix
}

\author{
David V. Bates
}

During the past five years, we have witnessed an avalanche of research on the adverse health effects of air pollutants. After the major visible pollution of smoke emission had been dealt with, and when controls were in place for reductions in emissions from coal burning, the industrial world was tempted to assume that the problem of air pollution, which had culminated in the London episode of December 1952, no longer existed. Of course, ozone formation was recognized to be a problem in Los Angeles. But, stimulated by "timeseries" studies of the daily levels of particulate pollution and the concurrent daily deaths or hospital admissions or emergency visits, we have learned that fine particles (less than 10 microns in size or $\mathrm{PM}_{10}$ ) are associated with increased mortality from respiratory and cardiovascular causes. They are also associated with increased hospital admissions for asthma, pneumonia, chronic obstructive lung disease, and cardiovascular conditions; with depressed lung function and increased respiratory symptoms in children; and possibly with significant changes in heart rate in the elderly.

It has been difficult to keep abreast of the many studies that have been published; but the consistency of these in places with very different populations, climates, and other concomitant pollutants, has led to the opinion of many observers that a causal relationship must be assumed, even in the absence of an understanding of the biological mechanism.

Canadian data from Toronto and Southern Ontario have contributed importantly to this information, and much of this has been work published by Health Canada. This issue of the Journal contains the most recent of these studies by Burnett and his colleagues (pages 152-56). They studied the daily deaths, excluding accidents and suicides, from 11 Canadian cities including all the major centres, with a total population of 9.85 million people, from 1980 to 1991. As one would expect, the pollutants measured, $\mathrm{CO}, \mathrm{NO}_{2}, \mathrm{SO}_{2}$, and $\mathrm{O}_{3}$, were highly inter-correlated. Daily measures of particle mass, such as $\mathrm{PM}_{10}$, were not available for analysis and could not be included in the multiple pollutant models.

The authors report that $\mathrm{NO}_{2}$ was the pollutant most strongly associated with daily mortality, with ozone, sulphur dioxide and carbon monoxide also significantly associated but less strongly. The authors have concluded that ambient air pollution "generated from the burning of fossil fuels is a risk factor for premature mortality in Canadian cities", and they suggest that their estimates can be used to determine public health benefits due to air pollution mitigation strategies. Their work confirms the many studies which have indicated that combustion products are currently responsible for a variety of adverse health effects. It is unfortunate that comprehensive $\mathrm{PM}_{10}$ data were not available for this study since it might have been found that this pollutant had a stronger association than ambient $\mathrm{NO}_{2}$, which, being an indicator of exposure to vehicle exhaust, might well be a surrogate for it.

There are a number of reasons suggesting that the observed outcome should not be directly attributed to $\mathrm{NO}_{2}$; in these low concentrations it seems unlikely that it could be having a direct effect. Furthermore, there have been recent examples in which $\mathrm{NO}_{2}$ was at far higher concentrations than are found in these
Canadian cities, but in which dramatic effects on mortality did not occur. In London, England, for example, in December 1991, $\mathrm{NO}_{2}$ reached a level of $400 \mathrm{ppb}$ for eight consecutive hours on two consecutive days. There was a small blip in mortality, but it seemed likely that this could be accounted for by the concomitant rise in $\mathrm{PM}_{10}$. This was not being measured at the time, but as visibility was obviously reduced in the episode, levels of over $200 \mathrm{micrograms} / \mathrm{m}^{3}$ were probable. The international literature, particularly the data coming from the ambitious European project known as APHEA, does contain some evidence of associations between various health outcomes and $\mathrm{NO}_{2}$, but the data are not consistent across different cities. One of the difficulties is that although exposure to indoor $\mathrm{NO}_{2}$ from gas cooking has been carefully quantified, we do not have much information on the general relationship between individual exposures and the results of ambient monitoring; what there is, from a study of schoolchildren in Denmark, suggests that outdoor monitors do reflect personal exposures reasonably faithfully.

The interest of the media in the adverse health effects of air pollutants at current levels, is ephemeral. Current data indicate that the public health community cannot be complacent about this problem; air monitoring should be not only maintained, but improved; and research, using modern large databases, should be continued. In the present climate of budget cuts, it is important to preserve the research capability in this field which the group at Health Canada has exploited with such distinction. 


\title{
Pollution atmosphérique : Le phoenix de l'environnement
}

\author{
David V. Bates
}

Au cours des cinq dernières années, nous avons été les témoins d'une avalanche de recherches portant sur les effets néfastes des polluants de l'air sur la santé. Après avoir éliminé la pollution très visible engendrée par les émissions de fumée, et une fois mis en place les moyens de contrôle visant à réduire les émissions résultant de la combustion du charbon, le monde industrialisé a tenu pour acquis que le problème de la pollution atmosphérique, qui a connu sa phase la plus aiguë en décembre 1952 à Londres, était désormais du passé. Certes, on se rendait compte que l'ozone faisait problème à Los Angeles. Mais, confrontés aux études chronologiques des niveaux quotidiens de pollution particulaire ainsi qu'en présence de décès, d'hospitalisations ou de visites à l'urgence survenant au quotidien, nous avons découvert que les fines particules (de moins de 10 microns ou $\mathrm{MP}_{10}$ ) sont associées à une plus grande mortalité causée par des troubles respiratoires et cardiovasculaires. Ces particules sont également associées à un plus grand nombre d'hospitalisations pour crises d'asthme, pneumonies, maladies respiratoires obstructives chroniques, et problèmes cardiovasculaires; à l'affaiblissement de la fonction pulmonaire et à l'augmentation des symptômes de troubles respiratoires chez les enfants; et peut-être à des changements importants du rythme cardiaque des personnes âgées.

Il est difficile de se tenir au courant de toutes les nombreuses études qui ont été publiées; mais la constance des résultats de ces études faites à différents endroits sur des populations et des climats très différents, ainsi que sur d'autres polluants concomitants, ont amené de nombreux observateurs à supposer une relation de cause à effet, même sans parfaitement comprendre le mécanisme biologique.

Professeur émérite de médecine, Département de soins de santé et épidémiologie, Université de la Colombie-Britannique, Mather Building, 5804 Fairview Ave., Vancouver, C.-B. V6T 1 Z3
Les données canadiennes concernant Toronto et le sud de l'Ontario ont beaucoup contribué à l'accumulation des informations dans ce domaine, dont la plupart sont le fruit de travaux publiés par Santé Canada. On trouvera dans ce numéro de la Revue les plus récentes de ces études faites par Burnett et ses collègues (aux pages 152-56). Ils ont étudié les cas de décès quotidiens, à l'exclusion des accidents et des suicides, dans 11 villes canadiennes dont les principaux centres urbains, regroupant une population totale de 9,85 millions de personnes, de 1980 à 1991 . Comme on pouvait s'y attendre, on a constaté une grande corrélation entre les polluants mesurés, nommément le $\mathrm{CO}$, le $\mathrm{NO}_{2}$, le $\mathrm{SO}_{2}$ et $\mathrm{l}^{\prime} \mathrm{O}_{3}$. On ne disposait pas des mesures quotidiennes de la masse de particule, comme la $\mathrm{MP}_{10}$, pour pouvoir les analyser et il a donc été impossible de les intégrer dans les modèles de polluants multiples.

Les auteurs déclarent que le $\mathrm{NO}_{2}$ est apparu comme le polluant le plus fortement associé à la mortalité quotidienne, l'ozone, le dioxide de soufre et le monoxide de carbone étant également significativement associés mais dans une moindre mesure. Les auteurs en ont conclu que la pollution atmosphérique ambiante, "générée par la combustion de carburants fossiles, constitue un facteur de risque de mortalité prématurée dans les villes canadiennes», et ils proposent que leurs estimations soient utilisées pour déterminer les avantages que peuvent offrir pour la santé publique les stratégies visant à atténuer la pollution atmosphérique. Leurs recherches corroborent les résultats de nombreuses études indiquant que les produits de combustion sont à l'heure actuelle responsables de tout un ensemble d'effets préjudiciables sur la santé. Il est regrettable que les données complètes relatives à la $\mathrm{MP}_{10}$ n'aient pas été disponibles pour cette étude, car elles auraient peut-être permis de découvrir que l'association de ce polluant était plus forte que celle du $\mathrm{NO}_{2}$ ambiant, lequel, étant un indicateur d'exposition aux gaz d'échappement des véhicules, pourrait bien lui servir de substitut.

Il y a plusieurs raisons qui portent à croire que les résultats observés ne peuvent pas être directement imputables au $\mathrm{NO}_{2}$; à ces faibles niveaux de concentration, il semble peu probable qu'il puisse avoir un effet direct. En outre, on a vu de récents exemples dans lesquels le $\mathrm{NO}_{2}$ était présent en beaucoup plus fortes concentrations que celles détectées dans les villes canadiennes, mais sans que l'on constate pour autant les mêmes résultats tragiques au niveau de la mortalité. Par exemple, en décembre 1991, à Londres, en Angleterre, la concentration de $\mathrm{NO}_{2}$ a atteint un niveau de 400 parties par milliard pendant huit heures consécutives, pendant deux jours d'affilée. On a observé une légère hausse de la mortalité mais il semble qu'elle puisse être expliquée par l'augmentation concomitante de la $\mathrm{MP}_{10}$. On ne l'a pas mesurée à l'époque, mais étant donné la réduction de la visibilité au cours de cet épisode, il est probable que les niveaux se situaient à plus de 200 $\mu \mathrm{g} / \mathrm{m}^{3}$. La littérature internationale, et tout particulièrement les données provenant de l'ambitieux projet européen connu comme l'APHEA, présente quelques éléments à l'appui d'associations entre les différents résultats de santé et le $\mathrm{NO}_{2}$, mais ces données ne sont pas uniformes d'une ville à l'autre. L'une des difficultés vient du fait que même si l'exposition au $\mathrm{NO}_{2}$ à l'intérieur résultant de la combustion du gaz de cuisson a été soigneusement quantifiée, nous ne disposons pas de beaucoup d'informations sur le rapport général existant entre les expositions individuelles et les résultats de la surveillance de l'air ambiant; le peu qui soit disponible provient d'une étude faite sur des enfants d'âge scolaire au Danemark qui montre que les détecteurs de pollution extérieure enregistrent assez fidèlement les expositions des individus.

L'intérêt manifesté par les médias pour les effets néfastes des polluants atmos- 
phériques sur la santé aux niveaux actuels ne sera que passager. Les données courantes indiquent que la communauté de santé publique ne peut pas se permettre d'ignorer ce problème; la surveillance de l'atmosphère doit non seulement être maintenue, mais il faut aussi l'améliorer; et les recherches à l'aide des grandes bases de données modernes doivent se poursuivre. Dans la conjoncture actuelle caractérisée par des coupes budgétaires, il est important de préserver les capacités de recherche dans ce domaine que l'équipe de Santé Canada est parvenue à si bien exploiter.

\section{In Memoriam}

Dr. Michael Davidson Nelson, age 51, passed away April 23, 1998, in Nanaimo, B.C. Mike was a loving and devoted husband and father, and deeply committed to friends and the communities in which he resided. As an applied sociologist, and a creative thinker with a passionate social conscience, Mike worked to make the world around him a better place in which to live. In the 1980s, he worked for four federal government departments, holding senior positions in policy, evaluation and research, and program development. After moving to British Columbia in 1991, Mike combined consulting and occasional university teaching with a high level of volunteer work in national and local organizations. At the time of his death, Mike headed NHN Consulting Group, was Vice-President of the Public Health Association of British Columbia, a director of the Mid-Island Public Participation Society, and Clinical Assistant Professor in the Institute for Health Promotion Research, University of British Columbia. He formerly served as a director of the Canadian Public Health Association (Chair of the Environmental and Occupational Health Division), Co-Chair of the City of Nanaimo's Social Planning Advisory Committee, and President of Manotick (Ontario) Project Ploughshares. He will be terribly missed by his wife, Dr. Susan Hess Nelson, son, Chad, daughter, Janet, parents, Dr. Charles and Dorothea Nelson, brothers William (Jacki) and James (Dr. Mary), aunt, Berniece Brown (Donald), in-laws (the Hess family), and many cousins, nephews and nieces. All who knew Mike are invited to share memories of his wisdom, passion, wit, sense of humour and enjoyment of life.

Le Dr Michael Davidson Nelson est décédé le 23 avril 1998, à l'âge de 51 ans, à Nanaimo (C.-B.). Mari et père affectueux et dévoué, Mike était fidèle à ses amis et profondément engagé dans les communautés où il a vécu. En tant que spécialiste de la sociologie appliquée, doté d'une pensée féconde et animé d'une profonde conscience sociale, Mike a toujours cherché à améliorer le monde autour de lui pour qu'il y fasse meilleur vivre. Dans les années 1980, il a travaillé dans quatre ministères du gouvernement fédéral où il a occupé de hautes fonctions dans les domaines de la politique, de l'évaluation et de la recherche, ainsi que dans l'élaboration de programmes. En 1991, il va s’installer en Colombie-Britannique où il devient consultant et enseignant occasionnel à l'université, en plus d'assumer bénévolement de multiples tâches au sein d'organismes nationaux et locaux. Au moment de sa mort, Mike était à la tête du NHN Consulting Group, vice-président de l'Association de santé publique de la Colombie-Britannique, et directeur de la Mid-Island Public Participation Society, ainsi que professeur adjoint d'enseignement clinique à l'Institut de recherche pour la promotion de la santé de l'Université de la ColombieBritannique. Il a aussi occupé un poste de directeur à l'Association canadienne de santé publique (président de la Division de l'environnement et de la santé et sécurité au travail), a été co-président du comité consultatif de planification sociale de la ville de Nanaimo et président du Manotick Project Ploughshares (Ontario). Nous ne doutons pas qu'il laissera un grand vide dans la vie de sa femme, le Dr Susan Hess Nelson, de son fils Chad, de sa fille Janet, de ses parents le Dr Charles et madame Dorothea Nelson, de ses frères William (Jacki) et James (Dr Mary), de sa tante Berniece Brown (Donald), de ses beaux-parents (la famille Hess), ainsi que de ses cousins, neveux et nièces. Tous ceux qui l'ont connu sont invités à se souvenir de sa sagesse, de sa passion, de sa vivacité d'esprit, de son sens de l'humour et du goût qu'il manifestait pour la vie. 